We find total cluster radii to be between 2 and $4 \mathrm{Mpc}$ and total cluster populations between 30 to some hundreds of galaxies to the limiting magnitude of our counts which is about one and a half magnitudes brighter than the plate limit.

From the projected number-density distribution we determined the radius of the sphere around the cluster centre which contains 20 galaxies. We found that this radius is about $0.3 \mathrm{Mpc}$ independent of the total population of the cluster if it contains more than 170 galaxies. In any case a value of $0.2 \mathrm{Mpc}$ is a lower limit for the radius of the sphere defined in this way. This corresponds to an upper limit for the mean number density in this sphere for all clusters.

Of course we have counted galaxies to different absolute magnitudes because of the different distances of the clusters, al1 of which are detected to the same limiting apparent magnitudes. This effect has little influence on the radii of the spheres containing 20 galaxies because of luminosity segregation in the centres of clusters of galaxies. But the total cluster characteristics will depend on these magnitude limits.

We will discuss this effect in detail in connection with a more extensive sample of clusters in a future paper. Our results obtained up to the present time are published in a series of papers in Astronomische Nachrichten.

\title{
MATHEMATICAL APPROACH TO THE PROBLEM OF CLUSTERING
}

J. Burczyk and A. Zieba

The mathematical theory of the description of the structure of point-sets in a plane or in space can be applied to the investigation of the distribution of different extragalactic objects. Mathematically we can define rigorously such ideas as cluster, nucleus of cluster, monoor polynuclear structure, background and so on. First we choose a critical distance that determines the scale on which we investigate the distribution of objects. The critical distance plays a similar role to the wavelength of radiation by means of which we investigate the internal structure of matter. It is obvious that the description is different for different critical distances. To obtain a complete picture we have to analyse the evolution of this description as the critical distance changes.

The mathematical theory of the description of the structure of point sets can be applied to investigations of individual clusters as well as to the statistical description of a family of clusters. We can characterize strictly the given distribution as well as compare different distributions.

If the point-set under under investigation is very numerous the 
description can be very complicated. In this case it is worth applying the method of statistical reduction. The main idea of the method of statistical reduction is the following: we replace the real population by a new, reduced population which is distributed according to the same rules as the real population. The process of reduction is a mathematical one based on the theory of probability. As a result of the reduction method we lose information but we gain on simplicity of description. We can lose much information but gain much simplicity and it depends on our choice. In every particular case we are able to regulate this choice in an optimal way. As we know, it is very difficult to compare the structures of two sets with different numbers of points. An additional important advantage of the method of statistical reduction is the fact that different sets after reduction can possess the same number of objects.

As the starting-point in describing the distribution of galaxies we must know the coordinates of galaxies or their numbers in squares by rectangular division of the map. In the first case the method is not only more accurate but also applicable to investigations of individual clusters. Applications to distribution of different extragalactic objects are in progress. In the second case we have already some statistical results. Professor Rudnicki will describe some of them.

\section{RADIO EMISSION OF ABELL CLUSTERS IN THE GB AND GB2 REGIONS}

Adam Michalec and Jerzy Machalski

The observational material is based upon pencil-beam surveys made with the NRAO 300-ft radio telescope at $1400 \mathrm{MHz}$. The GB survey (Maslowski 1972) covers $0.1586 \mathrm{sr}$ in the zone $7^{\mathrm{h}} 15^{\mathrm{m}} \leqslant \alpha \leqslant 16^{\mathrm{h}} 20^{\mathrm{m}}$, $459.9 \leqslant \delta<5198$. The GB2 survey made by Machalski in 1975 was carried out in the region defined by $7^{\mathrm{h}} 08^{\mathrm{m}} \leqslant \alpha \leqslant 16^{\mathrm{h}_{5}} 8^{\mathrm{m}}, 31.8 \leqslant \delta \leqslant 39.6$ (0.2828 sr).

The aim of this work is as follows:

(1) to identify the sources in Abe11 clusters,

(2) to estimate the luminosity distribution at $1400 \mathrm{MHz}$ and compare it with that of Owen (1975),

(3) to search for a dependence of spectra on luminosity.

We have obtained the following results:

Number of Abell clusters in the region

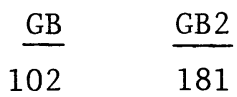

Number of detections within $0.3 \mathrm{r}_{\mathrm{c}}$

(cf. Wills 1966) with $\mathrm{S}_{1400} \geqslant 0.09 \mathrm{Jy}$; with $\mathrm{S}_{1400}<0.09 \mathrm{Jy}$

$\begin{array}{ll}31 * & 39 \\ \text { no data } & 12\end{array}$

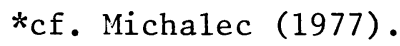

